NISSUNA UMANA INVESTIGAZIONE SI PUO DIMANDARE VERA SCIENZIA S'ESSA NON PASSA PER LE MATEMATICHE DIMOSTRAZIONI LEONARDO DA VINCI

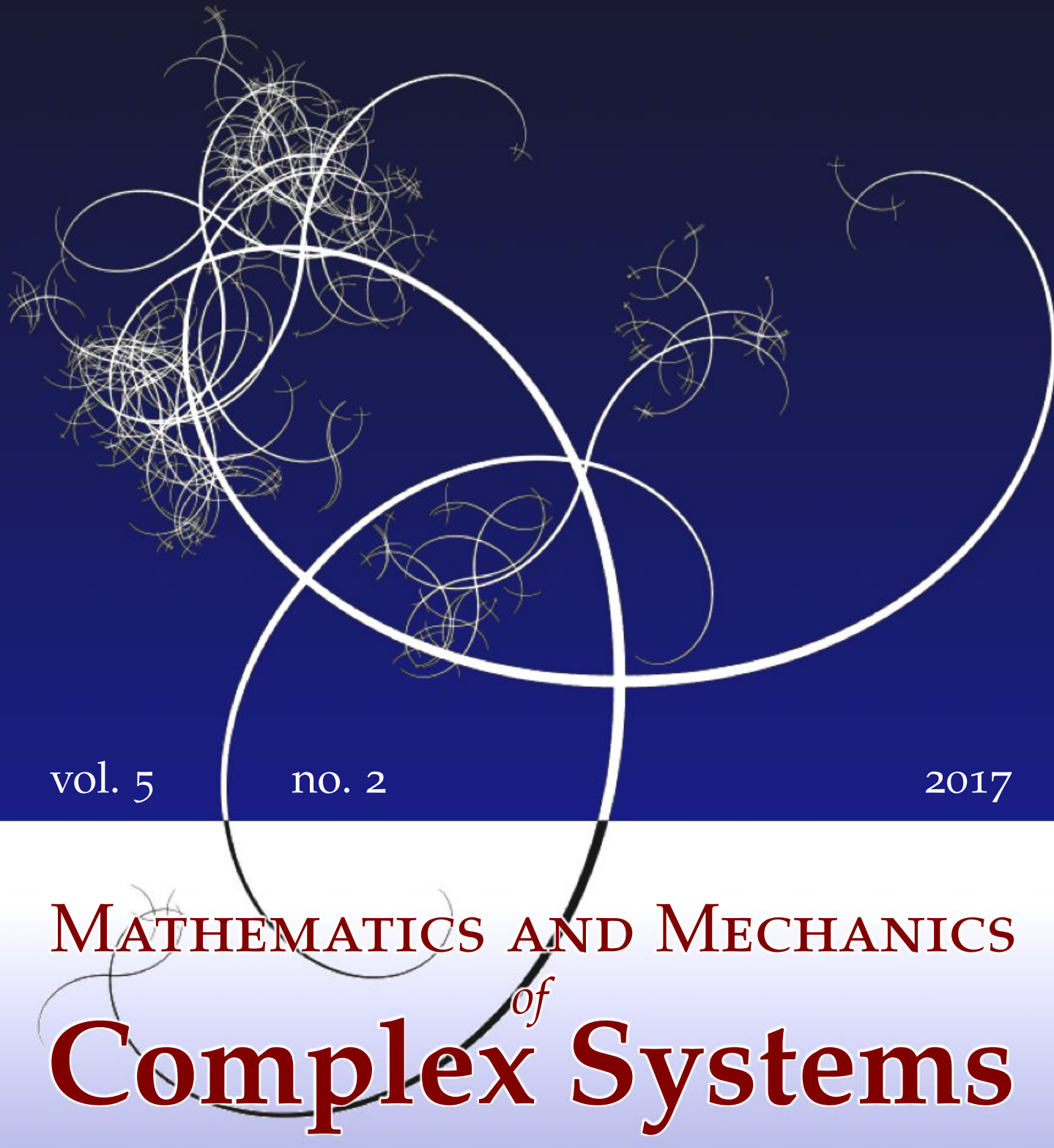

MiroslaV ŠILHAVÝ

THE GENERAL FORM OF THE RELAXATION OF A PURELY INTERFACIAL ENERGY FOR STRUCTURED DEFORMATIONS 


\title{
THE GENERAL FORM OF THE RELAXATION OF A PURELY INTERFACIAL ENERGY FOR STRUCTURED DEFORMATIONS
}

\author{
MiroslaV ŠILhaVÝ
}

\begin{abstract}
This paper deals with the relaxation of energies of media with structured deformations introduced by Del Piero and Owen (1993; 1995). Structured deformations provide a multiscale geometry that captures the contributions at the macrolevel of both smooth and nonsmooth geometrical changes (disarrangements) at submacroscopic levels. The paper examines the special case of Choksi and Fonseca's (1997) energetics of structured deformations in which the unrelaxed energy does not contain the bulk contribution. Thus, the energy is purely interfacial but of a general form. New formulas for the relaxed bulk and interfacial energies are proved. The bulk relaxed energy is shown to coincide with the subadditive envelope of the unrelaxed interfacial energy while the relaxed interfacial energy is the restriction of the envelope to rank-1 tensors. Moreover, it is shown that the minimizing sequence required to define the bulk energy in the relaxation scheme of Choksi and Fonseca (1997) can be realized in the more restrictive class required in the relaxation scheme of Baía, Matias and Santos (2012), thus establishing the equality of relaxed energies of the two approaches for general purely interfacial energies. The relaxations of the specific interfacial energies of Owen and Paroni (2015) and Barroso, Matias, Morandotti and Owen (2017) are simple consequences of our general results.
\end{abstract}

\section{Introduction}

This paper deals with the relaxation of nonclassical continua modeled as media with structured deformations introduced by Del Piero and Owen [1993; 1995]. ${ }^{1}$ In their original setting, a structured deformation is a triplet $(\mathcal{K}, g, G)$ of objects of the following nature. The set $\mathcal{K} \subset \boldsymbol{R}^{3}$, the crack site, is a subset of vanishing Lebesgue measure of the reference region $\Omega$, the map $g: \Omega \backslash \mathcal{K} \rightarrow \boldsymbol{R}^{3}$, the deformation map, is piecewise continuously differentiable and injective, and $G$ is a piecewise

\section{Communicated by Gianpietro Del Piero.}

MSC2010: primary 49J45; secondary 74A60, 74G65, 15A99.

Keywords: structured deformations, relaxation, subadditive envelope, interfacial energy, bulk energy, functions of measures.

${ }^{1}$ The reader is referred to the proceedings [Del Piero and Owen 2004] and to the recent survey [Baía et al. 2011] for additional references and for further developments. 
continuous map from $\Omega \backslash \mathcal{K}$ to the set of invertible second-order tensors describing deformation without disarrangements.

Within this context, simple deformations are triples $(\mathcal{K}, g, \nabla g)$ where $g$ is a piecewise smooth injective map with jump discontinuities describing partial or full separation of pieces of the body. In view of this, in the general case of a structured deformation $(\mathcal{K}, g, G)$, the tensor

$$
H=\nabla g-G,
$$

the deformation due to disarrangements, measures the departure of $(\mathcal{K}, g, G)$ from the simple deformation $(\mathcal{K}, g, \nabla g)$.

Choksi and Fonseca [1997] introduced into the theory of structured deformations energy considerations and the ideas of relaxation. For further studies in one- and multidimensional settings, see [Del Piero 2001; 2004]. It is well-known that the existing techniques of relaxation of the calculus of variations and continuum mechanics are unable to cope with injectivity requirements. Accordingly, Choksi and Fonseca neglect the injectivity requirement; in addition, they assume weaker regularity. In their interpretation, structured deformations are pairs $(g, G)$ where $g: \Omega \rightarrow \boldsymbol{R}^{n}$ is a special $\boldsymbol{R}^{n}$-valued map of bounded variation from the space $S B V(\Omega)$ and $G: \Omega \rightarrow$ Lin is an integrable Lin-valued map from the space $\mathrm{七}^{1}(\Omega$, Lin $) .^{2}$ Thus,

$$
S D(\Omega):=S B V(\Omega) \times \longleftarrow^{1}(\Omega, \text { Lin })
$$

is the set of all structured deformations. Structured deformations of the form $(g, \nabla g)$ with $g \in S B V(\Omega)$ are called simple deformations in this paper.

The relaxation starts from the energy

$$
E(g)=\int_{\Omega} W(\nabla g) d \mathcal{V}+\int_{J(g)} \psi\left(\llbracket g \rrbracket, v_{g}\right) d \mathcal{A}
$$

of a simple deformation $g \in S B V(\Omega)$. Here $\mathcal{V}$ and $\mathcal{A}$ are the Lebesgue measure and the $(n-1)$-dimensional Hausdorff measure in $\boldsymbol{R}^{n}$ and $\nabla g$ is the absolutely continuous part of the derivative (= gradient) $\mathrm{D} g$ of $g$, while the singular part

$$
\mathrm{D}^{\mathrm{s}} g:=\llbracket g \rrbracket \otimes v_{g} \mathcal{A}\llcorner J(g)
$$

is a tensor-valued singular measure describing the discontinuities of $g$; that part is formed from the jump set $J(g) \subset \Omega$ of $g$, the jump $\llbracket g \rrbracket$ of $g$ on $J(g)$ and the normal $v_{g}$ to $J(g)$. The reader is referred to (24), below, for a detailed description of these objects. The material is characterized by the bulk energy density $W: \operatorname{Lin} \rightarrow \boldsymbol{R}$ and

${ }^{2}$ For brevity of notation, we omit the target spaces and write $S B V(\Omega) \equiv S B V\left(\Omega, \boldsymbol{R}^{n}\right)$ and $\mathrm{七}^{1}(\Omega$, Lin $) \equiv L^{1}(\Omega$, Lin $)$. See Section 3 for more notation and detailed definitions. 
by the interfacial (or cohesion) energy $\psi: \boldsymbol{D}_{n} \rightarrow \boldsymbol{R}$, where we denote

$$
\boldsymbol{D}_{n}=\boldsymbol{R}^{n} \times \boldsymbol{S}^{n-1} .
$$

The approximation theorem of Del Piero and Owen [1993, Theorem 5.8] says that every structured deformation is a well-defined limit of simple deformations. In the framework of Choksi and Fonseca [1997] (see also [Šilhavý 2015]), this means that corresponding to each structured deformation $(g, G) \in S D(\Omega)$ there exists a sequence $\left(g_{k}, \nabla g_{k}\right) \in S D(\Omega)$ (i.e., with $g_{k}$ in $\left.S B V(\Omega)\right)$ such that

$$
\begin{gathered}
g_{k} \rightarrow g \quad \text { in } \longleftarrow^{1}\left(\Omega, \boldsymbol{R}^{n}\right), \\
\nabla g_{k} \rightarrow^{*} G \quad \text { in } \mathcal{M}(\Omega, \text { Lin }), \\
\sup \left\{\left|\nabla g_{k}\right|_{\bigsqcup^{1}\left(\Omega, \boldsymbol{R}^{n}\right)}: k=1, \ldots\right\}<\infty .
\end{gathered}
$$

The relaxed energy of a structured deformation $(g, G) \in S D(\Omega)$ is defined by

$$
I(g, G)=\inf \left\{\liminf _{k \rightarrow \infty} E\left(g_{k}\right): g_{k} \in S B V(\Omega) \text { satisfies }(2)\right\} .
$$

Thus, a sequence approaching the above infimum realizes the most economical way to build up the deformation $(g, G)$ using approximations in $S B V$. The relaxation theorem of Choksi and Fonseca [1997, Theorems 2.6 and 2.17, Remark 3.3] says that, under some assumptions on $W$ and $\psi$ (a particular case of which are Assumptions 2.1, below), the relaxed energy admits the integral representation

$$
I(g, G)=\int_{\Omega} H(\nabla g, G) d \mathcal{V}+\int_{J(g)} h\left(\llbracket g \rrbracket, v_{g}\right) d \mathcal{A}
$$

where $H$ and $h$ are some functions determined explicitly in the cited theorems (Theorem 2.2 presents formulas for $H$ and $h$ for a particular case).

This paper deals with the relaxation of energy functions $E$ for which the bulk contribution vanishes, i.e., with energy functions of the form

$$
E(g)=\int_{J(g)} \psi\left(\llbracket g \rrbracket, v_{g}\right) d \mathcal{A}
$$

for each $g \in S B V(\Omega)$. The main result, Theorem 2.3, below, gives explicit descriptions of the functions $H$ and $h$ from (4) and applies them to give simplified proofs of two particular cases Examples 2.5 and 2.6 given previously in [Owen and Paroni 2015; Barroso et al. 2017].

\section{The main result and examples}

We make the following standing hypotheses about $\psi$.

2.1. Assumptions. (i) The function $\psi: \boldsymbol{D}_{n} \rightarrow \boldsymbol{R}$ is continuous. 
(ii) We have $\psi(-a,-b)=\psi(a, b)$ and

$$
0 \leq \psi(a, b) \leq C_{1}|a|
$$

for every $(a, b) \in \boldsymbol{D}_{n}$ and some $C_{1}>0$.

(iii) The function $\psi(\cdot, v)$ is subadditive and positively homogeneous for each $v \in S^{n-1}$.

To ease the statements of the results, we extend any function $\zeta: \boldsymbol{D}_{n} \rightarrow[0, \infty)$ to an identically denoted function $\zeta: \boldsymbol{R}^{n} \times \boldsymbol{R}^{n} \rightarrow[0, \infty)$ by homogeneity with respect to the second variable, i.e., by assuming that the extended function satisfies

$$
\zeta(a, t b)=t \zeta(a, b)
$$

for any $t \geq 0$ and $(a, b) \in \boldsymbol{R}^{n} \times \boldsymbol{R}^{n}$. This convention applies in particular to the functions $\psi$ and $h$.

We need some notation to formulate the main results. Let $Q=\left(-\frac{1}{2}, \frac{1}{2}\right)^{n}$, and for every $M \in \operatorname{Lin}$, let $w_{M}: \partial Q \rightarrow \boldsymbol{R}^{n}$ be given by

$$
w_{M}(x)=M x \quad \text { for every } x \in \partial Q \text {. }
$$

Furthermore, if $(a, b) \in \boldsymbol{D}_{n}$, let $Q_{b}$ be any cube with unit edge, center at $0 \in \boldsymbol{R}^{n}$ and two faces normal to $b$, and let $z_{a, b}: Q_{b} \rightarrow \boldsymbol{R}^{n}$ be the map defined by

$$
z_{a, b}(x)=\frac{1}{2} a(\operatorname{sgn}(x \cdot b)+1), \quad x \in Q_{b} .
$$

Finally, if $u \in S B V(\Omega)$, let us put

$$
\Psi\left(\mathrm{D}^{\mathrm{s}} u\right):=\int_{J(u)} \psi\left(\llbracket u \rrbracket, v_{u}\right) d \mathcal{A} .
$$

The following statement is a particular case $W=0$ of the relaxation theorem of Choksi and Fonseca [1997, Theorems 2.6 and 2.17, Remark 3.3].

2.2. Theorem. The effective energies $H$ and $h$ are given by

$$
H(A, B)=\inf \left\{\Psi\left(\mathrm{D}^{\mathrm{s}} u\right): u \in S B V(Q), u=w_{A} \text { on } \partial Q, \int_{Q} \nabla u d \mathcal{V}=B\right\}
$$

for each $A, B \in$ Lin and

$$
h(a, b)=\inf \left\{\Psi\left(\mathrm{D}^{\mathrm{s}} u\right): u \in \operatorname{SBV}\left(Q_{b}\right), u=z_{a, b} \text { on } \partial Q_{b}, \nabla u=0 \text { on } Q_{b}\right\}
$$

for each $(a, b) \in \boldsymbol{D}_{n}$.

The following theorem, the main result of this paper, shows that the functions $H$ and $h$ admit a much more explicit description in terms of a single function $\Phi$. 
2.3. Theorem. The functions $H$ and $h$ in Theorem 2.2 are given by

$$
\begin{gathered}
H(A, B)=\Phi(A-B), \\
h(a, b)=\Phi(a \otimes b)
\end{gathered}
$$

for every $A, B \in \operatorname{Lin}$ and $(a, b) \in \boldsymbol{D}_{n}$, where $\Phi$ is a subadditive and positively homogeneous function on Lin defined by each of the following equivalent Assertions (i)-(iv); moreover, for dyadic arguments, we have an additional Assertion (v).

(i) $\Phi$ is the biggest subadditive function on Lin satisfying

$$
\Phi(a \otimes b) \leq \psi(a, b) \quad \text { for every }(a, b) \in \boldsymbol{D}_{n} ;
$$

i.e.,

$\Phi(M)=\sup \{\Theta(M): \Theta$ is subadditive on Lin

$$
\text { and } \left.\Theta(a \otimes b) \leq \psi(a, b) \text { for every }(a, b) \in \boldsymbol{D}_{n}\right\} \text {. }
$$

(ii) For every $M \in$ Lin, $^{3}$

$$
\Phi(M)=\inf \left\{\sum_{i=1}^{m} \psi\left(a_{i}, b_{i}\right):\left(a_{i}, b_{i}\right) \in \boldsymbol{D}_{n}, i=1, \ldots, m, \sum_{i=1}^{m} a_{i} \otimes b_{i}=M\right\} .
$$

(iii) For every $M \in$ Lin,

$$
\Phi(M)=\inf \left\{\Psi\left(\mathrm{D}^{\mathrm{s}} u\right): u \in S B V(Q), u=w_{M} \text { on } \partial Q, \nabla u=0 \text { on } Q\right\} .
$$

(iv) For every $M \in$ Lin,

$$
\Phi(M)=\inf \left\{\Psi\left(\mathrm{D}^{\mathrm{s}} u\right): u \in S B V(Q), u=w_{M} \text { on } \partial Q, \int_{Q} \nabla u d \mathcal{V}=0\right\} .
$$

(v) For arguments of the form $a \otimes b$, where $(a, b) \in \boldsymbol{D}_{n}$, $\Phi(a \otimes b)=\inf \left\{\Psi\left(\mathrm{D}^{\mathrm{s}} u\right): u \in \operatorname{SBV}\left(Q_{b}\right), u=z_{a, b}\right.$ on $\partial Q_{b}, \nabla u=0$ on $\left.Q_{b}\right\}$.

The proof of Theorem 2.3 is given in Sections 5 and 6, below.

2.4. Remarks. (a) Since the pointwise supremum of any family of subadditive functions is subadditive (e.g., [Hille and Phillips 1957, Theorem 7.2.2]), (15) really defines a subadditive function.

(b) Among the above characterizations of $\Phi$, the closely related novel forms (i) and (ii) must be considered as the most important. The main advantage of (i) and (ii) is that they establish connections to the wealth of results of the convexity theory. These will be employed to analyze the examples to be formulated below.

3 Throughout the paper, the letter $m$ denotes any positive integer. 
(c) In one dimension, one can orient the normals to jumps to be always the vector +1 (rather than -1 ) and hence the dependence of $\psi$ on the second variable can be suppressed: $\psi=\psi(a), a \in \boldsymbol{R}$. Assumption 2.1(iii) then says that $\psi$ is subadditive and positively homogeneous. Thus, the subadditive envelope $\Phi$ of $\psi$ is $\psi$ itself, and all mentions of a subadditive envelope can be avoided. This is not the case if Assumption 2.1(iii) is relaxed. Indeed, working in one dimension, Del Piero [2001; 2004] calculated the relaxation of the energy (1) with the interfacial energy $\psi$ of a general form, avoiding Assumption 2.1(iii). His main result contains the subadditive envelope of $\psi$ also. In light of the above discussion, this envelope plays a different but related role. The relaxation of a purely interfacial energy of a more general form than that postulated in Assumptions 2.1 in arbitrary dimension will be treated in a future paper.

(d) The expressions in (iii)-(v) already occurred previously, albeit without noting that they are mutually equivalent and equivalent to (i) and (ii), except for some particular cases to be mentioned below. The formula for $H$ in (13a) with $\Phi$ defined in (iv) and the formula for $h$ in (13b) with $\Phi$ defined in (v) are direct consequences of Choksi and Fonseca's expressions in (11) and (12). The formula for $H$ with $\Phi$ given by (iii) crops up in the relaxation schemes by Baía, Matias and Santos [Baía et al. 2012, (3.2)] and by Barroso, Matias, Morandotti and Owen [Barroso et al. 2017, Theorem 3.2]. The relaxation schemes in the last two papers require among other things higher regularity of structured deformations and are not strictly comparable with that of Choksi and Fonseca described above.

(e) The infimum (iii) could be, in principle, bigger than (iv). Nevertheless, the infima are generally the same. This has been established previously in [Barroso et al. 2017] for the special choices of $\psi$ described in the following examples, which motivated the present study.

2.5. Example [Owen and Paroni 2015, Theorem 4, particular case $L=\mathrm{I}$ ]. If

$$
\psi_{|\cdot|}(a, b)=|a \cdot b| \quad \text { and } \quad \psi_{ \pm}(a, b)=\{a \cdot b\}_{ \pm}
$$

for every $(a, b) \in \boldsymbol{D}_{n}$, where $\{\cdot\}_{+}$and $\{\cdot\}_{-}$denote the positive and negative parts of a real number, then

$$
\begin{aligned}
\Phi_{|\cdot|}(M) & =|\operatorname{tr} M|, \\
\Phi_{ \pm}(M) & =\{\operatorname{tr} M\}_{ \pm}
\end{aligned}
$$

for every $M \in$ Lin. The effective energies $H_{|\cdot|}, H_{ \pm}, h_{|\cdot|}$ and $h_{ \pm}$are determined through $\Phi_{|\cdot|}$ and $\Phi_{ \pm}$by (13).

As shown in [Owen and Paroni 2015], $\{\operatorname{tr} M\}_{+}$is a volume density of disarrangements due to submacroscopic separations, $\{\operatorname{tr} M\}_{-}$is a volume density of 
disarrangements due to submacroscopic switches and interpenetrations, and $|\operatorname{tr} M|$ is a volume density of all three of these nontangential disarrangements: separations, switches and interpenetrations. The evaluation in [Owen and Paroni 2015] of $H$ (equivalently, of $\Phi$ ) for (21) is rather complicated; a recent paper by Barroso, Matias, Morandotti and Owen [Barroso et al. 2017] presents some simplification and the realization of the minimizing sequence in the narrower class (iv) in Theorem 2.3 mentioned earlier. Our version of the derivation, which includes the minimizing sequence from (iv) via Theorem 2.3 also, is given in Section 7.

2.6. Example [Barroso et al. 2017, (5.3)]. If

$$
\psi(a, b)=|a \cdot p|
$$

for $(a, b) \in \boldsymbol{D}_{n}$, where $p \in \boldsymbol{R}^{n}$ is a fixed vector, then

$$
\Phi(M)=\left|M^{\mathrm{T}} p\right|
$$

for any $M \in \operatorname{Lin}$.

\section{Notation and functions of bounded variation}

We denote by $\boldsymbol{Z}$ the set of integers, by $\boldsymbol{N}$ the set of positive integers, by $\boldsymbol{S}^{n-1}$ the unit sphere in $\boldsymbol{R}^{n}$ and by Lin the set of all linear transformations from $\boldsymbol{R}^{n}$ into itself, often identified with the set of $n \times n$ matrices with real elements. We use the symbols "." and " $|\cdot|$ " to denote the scalar product and the euclidean norm on $\boldsymbol{R}^{n}$ and on Lin. The latter are defined by $A \cdot B:=\operatorname{tr}\left(A B^{\mathrm{T}}\right)$ and $|A|=\sqrt{A \cdot A}$ where $A^{\mathrm{T}} \in \mathrm{Lin}$ is the transpose of $A$ and tr denotes the trace.

A real-valued function $f$ defined on a vector space $\mathrm{X}$ is said to be subadditive if $f(x+y) \leq f(x)+f(y)$ for every $x, y \in \mathrm{X}$ and positively homogeneous if $f(t x)=t f(x)$ for every $t \geq 0$ and $x \in \mathrm{X}$.

If $\Omega$ is an open subset of $\boldsymbol{R}^{n}$, we denote by $\longleftarrow^{1}(\Omega$, Lin) the space of Lin-valued integrable maps on $\Omega$. We denote by $\mathcal{M}(\Omega$, Lin) the set of all (finite) Lin-valued measures on $\Omega$. If $\mu \in \mathcal{M}(\Omega, \operatorname{Lin})$, we denote by $\mu\llcorner B$ the restriction of $\mu$ to a Borel set $B \subset \Omega$. If $G, G_{k} \in \mathrm{t}^{1}(\Omega, \mathrm{Lin}), k=1,2, \ldots$, we say that $G_{k}$ converges to $G$ in the sense of measures, and write

$$
G_{k} \rightarrow^{*} G \text { in } \mathcal{M}(\Omega, \text { Lin }),
$$

if $\int_{\Omega} G_{k} \cdot H d \mathcal{V} \rightarrow \int_{\Omega} G \cdot H d \mathcal{V}$ for every continuous map $H: \boldsymbol{R}^{n} \rightarrow$ Lin which vanishes outside $\Omega$.

We state some basic definitions and properties of the space $B V(\Omega)=B V\left(\Omega, \boldsymbol{R}^{n}\right)$ of maps of bounded variation and of the space $S B V(\Omega)=S B V\left(\Omega, \boldsymbol{R}^{n}\right)$, special maps of bounded variation. For more details, see [Ambrosio et al. 2000; Evans and Gariepy 1992; Ziemer 1989; Federer 1969]. 
We define the set $B V(\Omega)$ as the set of all $u \in L^{1}(\Omega)=L^{1}\left(\Omega, \boldsymbol{R}^{n}\right)$ such that there exists a measure $\mathrm{D} u \in \mathcal{M}(\Omega$, Lin) satisfying

$$
\int_{\Omega} u \cdot \operatorname{div} T d \mathcal{V}=-\int_{\Omega} T \cdot d \mathrm{D} u
$$

for each infinitely differentiable map $T: \boldsymbol{R}^{n} \rightarrow \boldsymbol{R}^{n \times n}$ which vanishes outside some compact subset of $\Omega$. Here $\operatorname{div} T$ is an $\boldsymbol{R}^{n}$-valued map on $\Omega$ given by $(\operatorname{div} T)_{i}=$ $\sum_{j=1}^{n} T_{i j, j}$, where the comma followed by an index $j$ denotes the partial derivative with respect to $j$-th variable. The measure $\mathrm{D} u$ is uniquely determined and called the weak (or generalized) derivative of $u$. We shall need the following form of the Gauss-Green theorem for $B V$ : if $\Omega$ is a domain with lipschitzian boundary and $u \in B V(\Omega)$, then there exists an $\mathcal{A}$ integrable map $u^{\partial \Omega}: \partial \Omega \rightarrow \boldsymbol{R}^{n}$ such that

$$
\mathrm{D} u(\Omega) \equiv \int_{\Omega} d \mathrm{D} u=\int_{\partial(\Omega)} u^{\partial \Omega} \otimes v_{\Omega} d \mathcal{A},
$$

where $v_{\Omega}$ is the outer normal to $\partial \Omega$. The map $u^{\partial \Omega}$ is determined within a change on a set of $\mathcal{A}$ measure 0 and is called the trace of $u$.

We define the set $\operatorname{SBV}(\Omega)$ as the set of all $u \in B V(\Omega)$ for which $\mathrm{D} u$ has the form

$$
\mathrm{D} u=\nabla u \mathcal{V}\left\llcorner\Omega+\llbracket u \rrbracket \otimes v_{u} \mathcal{A}\llcorner J(u)\right.
$$

where $\nabla u$, the absolutely continuous part of $\mathrm{D} u$, is a map in $亡^{1}(\Omega$, Lin) and the term

$$
\mathrm{D}^{\mathrm{s}} u:=\llbracket u \rrbracket \otimes v_{u} \mathcal{A}\llcorner J(u)
$$

on the right-hand side of (24) is called the jump (or singular) part of Du. The objects $J(u) \subset \Omega, \llbracket u \rrbracket: J(u) \rightarrow \boldsymbol{R}^{n}$ and $v_{u}: J(u) \rightarrow \boldsymbol{S}^{n-1}$ are called the jump set of $u$, the jump of $u$ and the normal to $J(u)$, respectively. Here $J(u)$ is the set of all $x \in \Omega$ for which there exist $v_{u}(x) \in \boldsymbol{S}^{n-1}$ and $u^{ \pm}(x) \in \boldsymbol{R}^{n}$ such that we have the approximate limits

$$
u^{ \pm}(x)=\operatorname{ap}_{\substack{y \rightarrow x \\ y \in \boldsymbol{H}^{ \pm}\left(x, v_{u}(x)\right)}} u(x)
$$

where $\boldsymbol{H}^{ \pm}\left(x, v_{u}(x)\right)=\left\{y \in \boldsymbol{R}^{n}: \pm(y-x) \cdot v_{u}(x)>0\right\}$. For a given $x \in \Omega$, either the triplet $\left(v_{u}, u^{+}, u^{-}\right)=\left(v_{u}(x), u^{+}(x), u^{-}(x)\right)$ does not exist or it is uniquely determined to within the change $\left(v_{u}, u^{+}, u^{-}\right) \mapsto\left(-v_{u}, u^{-}, u^{+}\right)$. With one of these choices, one puts $\llbracket u \rrbracket=u^{+}-u^{-}$and notes that $\llbracket u \rrbracket \otimes v_{u}$ is unique.

Finally, we denote by $\langle r\rangle$ the integral part of $r \in \boldsymbol{R}$. Clearly,

$$
\begin{aligned}
& r-1 \leq\langle r\rangle \leq r, \\
& 0 \leq r-\langle r\rangle \leq 1 .
\end{aligned}
$$


Writing $r=k t$, where $t \in \boldsymbol{R}$ and $k>0$, and dividing by $k$, we obtain

$$
0 \leq t-\langle k t\rangle / k \leq 1 / k
$$

and hence

$$
\langle k t\rangle / k \rightarrow t \quad \text { as } k \rightarrow \infty
$$

uniformly in $t \in \boldsymbol{R}$.

\section{Preliminary results}

We put

$$
\begin{aligned}
& \mathcal{C}(M):=\left\{u \in \operatorname{SBV}(Q): u=w_{M} \text { on } \partial Q, \nabla u=0 \text { on } Q\right\}, \\
& \mathcal{B}(M):=\left\{u \in \operatorname{SBV}(Q): u=w_{M} \text { on } \partial Q, \int_{Q} \nabla u d \mathcal{V}=0\right\}
\end{aligned}
$$

for any $M \in$ Lin. We start with the following preliminary results.

4.1. Proposition. If $A, B \in \operatorname{Lin}$ and $u \in \mathcal{B}(A)$ and $v \in \mathcal{B}(B)$, then $u+v \in \mathcal{B}(A+B)$ and

$$
\Psi\left(\mathrm{D}^{\mathrm{s}} u+\mathrm{D}^{\mathrm{s}} v\right) \leq \Psi\left(\mathrm{D}^{\mathrm{s}} u\right)+\Psi\left(\mathrm{D}^{\mathrm{s}} v\right)
$$

if $(J(u) \cap J(v))=0$, then we have the equality sign in (28).

Proof. We have

$$
J(u+v)=K_{u} \cup K_{v} \cup L
$$

where

$$
L=J(u) \cap J(v), \quad K_{u}=J(u) \backslash K, \quad K_{v}=J(v) \backslash K .
$$

Next, we observe that on $L$ we have $v_{u}(x)= \pm v_{v}(x)$ for $\mathcal{A}$-almost every $x \in L$; since we have a freedom in the choice of the sign of $v_{v}$, we assume $v_{u}(x)=v_{v}(x)$ and denote $\mu=v_{u}$ on $L$. Then

$$
[u+v] \otimes v_{u+v}= \begin{cases}{[u] \otimes v_{u}} & \text { on } K_{u}, \\ {[v] \otimes v_{v}} & \text { on } K_{v}, \\ ([u]+[v]) \otimes \mu & \text { on } L .\end{cases}
$$

By the subadditivity of $\psi$,

$$
\psi([u]+[v], \mu) \leq \psi([u], \mu)+\psi([v], \mu)=\psi\left([u], v_{u}\right)+\psi\left([v], v_{v}\right)
$$

and hence (30) provides

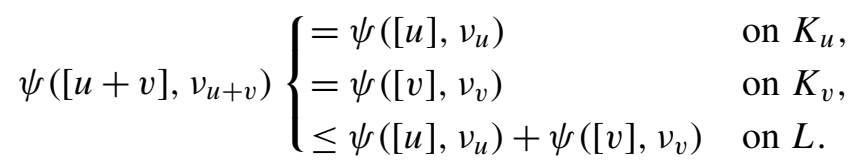


Integrating over $J(u+v)$ and using (29), we obtain

$$
\begin{aligned}
\Psi\left(\mathrm{D}^{\mathrm{s}} u+\mathrm{D}^{\mathrm{s}} v\right) & =\int_{J(u+v)} \psi\left([u+v], v_{u+v}\right) d \\
& \leq \int_{K_{u}} \psi\left([u], v_{u}\right) d+\int_{K_{v}} \psi\left([v], v_{v}\right) d \\
& \quad+\int_{L} \psi\left([u], v_{u}\right) d+\int_{L} \psi\left([v], v_{v}\right) d \\
& =\Psi\left(\mathrm{D}^{\mathrm{s}} u\right)+\Psi\left(\mathrm{D}^{\mathrm{s}} v\right),
\end{aligned}
$$

which completes the proof of (28).

4.2. Remark. If the interfacial energy density $\psi$ has the special form

$$
\psi(a, b)=\Lambda(a \otimes b)
$$

where $\Lambda: \operatorname{Lin} \rightarrow[0, \infty)$ is a subadditive and positively homogeneous function, then $\Psi\left(\mathrm{D}^{\mathrm{s}} u\right)$ is given by

$$
\Psi\left(\mathrm{D}^{\mathrm{s}} u\right)=\Lambda\left(\mathrm{D}^{\mathrm{s}} u\right)
$$

where $\mathrm{D}^{\mathrm{s}} u:=\llbracket u \rrbracket \otimes v_{u}\llcorner J(u)$ is the singular part of the derivative $\mathrm{D} u$ of $u$ and

$$
\Lambda\left(\mathrm{D}^{\mathrm{s}} u\right):=\int_{J(u)} \Lambda\left(\llbracket u \rrbracket \otimes v_{u}\right) d
$$

is an instance of Reshetnyak's [1968] functional $\mu \mapsto \Lambda(\mu)$ of a measure $\mu \in$ $\mathcal{M}(Q$, Lin $)$; see, e.g., [Ambrosio et al. 2000, (2.29)]. The subadditivity and positive homogeneity of $\Phi$ (asserted in Theorem 2.3) is then an instance of the general result [Ambrosio et al. 2000, Proposition 2.37] asserting the same properties of the functional $\mu \mapsto \Lambda(\mu)$. Indeed, if $M_{i} \in$ Lin and $u_{i} \in \mathcal{A}\left(M_{i}\right), i=1,2$, then $u_{1}+u_{2} \in \mathcal{A}\left(M_{1}+M_{2}\right)$ and therefore

$$
\Phi\left(M_{1}+M_{2}\right) \leq \Lambda\left(\mathrm{D}^{\mathrm{s}}\left(u_{1}+u_{2}\right)\right)=\Lambda\left(\mathrm{D}^{\mathrm{s}} u_{1}+\mathrm{D}^{\mathrm{s}} u_{2}\right) \leq \Lambda\left(\mathrm{D}^{\mathrm{s}} u_{1}\right)+\Lambda\left(\mathrm{D}^{\mathrm{s}} u_{2}\right)
$$

taking the infimum over all $u_{1} \in \mathcal{A}\left(M_{1}\right)$ and $u_{2} \in \mathcal{A}\left(M_{2}\right)$ gives

$$
\Phi\left(M_{1}+M_{2}\right) \leq \Phi\left(M_{1}\right)+\Phi\left(M_{2}\right) .
$$

The positive homogeneity follows similarly. We note that the interfacial energies in Examples 2.5 and 2.6 have the form (31), but this is not the case generally.

The following elementary result records some formulas to be employed below.

4.3. Remark. Let $\Omega \subset \boldsymbol{R}^{n}$ be an open bounded set with lipschitzian boundary. A countable family $\Omega_{\alpha}, \alpha \in N$, of pairwise disjoint subsets of $\Omega$ with lipschitzian boundaries is said to be a partition of $\Omega$ if one can write $\Omega=\bigcup_{\alpha=1}^{\infty} \Omega_{\alpha}$ to within a set of null Lebesgue measure. Let us agree to say that $\varphi \in L^{1}(\Omega, \boldsymbol{R})$ is piecewise constant if there exists a partition $\Omega_{\alpha}$ such that $\varphi$ is constant on each $\Omega_{\alpha}$. If $v_{\alpha}$ is 
the outer normal to $\Omega_{\alpha}$ and if $a_{\alpha}$ is the value of $\varphi$ on $\Omega_{\alpha}$, then $\varphi \in B V(\Omega, \boldsymbol{R})$ if and only if

$$
\sum_{(\alpha, \beta) \in \mathcal{I}} \int_{\partial \Omega_{\alpha} \cap \partial \Omega_{\beta}}\left|a_{\alpha}-a_{\beta}\right| d \mathcal{A}<\infty,
$$

where

$$
\mathcal{I}=\left\{(\alpha, \beta) \in N^{2}: \alpha<\beta,\left(\partial \Omega_{\alpha} \cap \partial \Omega_{\beta}\right)>0\right\} .
$$

If this is the case, we have the formulas

$$
\begin{aligned}
J(\varphi) & =\bigcup_{(\alpha, \beta) \in \mathcal{I}}\left(\partial \Omega_{\alpha} \cap \partial \Omega_{\beta}\right), \\
\llbracket \varphi \rrbracket v_{\varphi} & =\left(a_{\alpha}-a_{\beta}\right) v_{\beta} \quad \text { on } \partial \Omega_{\alpha} \cap \partial \Omega_{\beta} \text { for any }(\alpha, \beta) \in \mathcal{I}, \\
\mathrm{D} \varphi & =\llbracket \varphi \rrbracket v_{\varphi}\llcorner J(\varphi)
\end{aligned}
$$

to within changes on sets of null $\mathcal{A}$ measure. The total variation (mass) $\mathrm{M}(\mathrm{D} \varphi)$ of $\mathrm{D} \varphi$ is equal to the sum in (32).

Proof. Assume that (32) holds, and prove that $\varphi \in B V(\Omega, \boldsymbol{R})$ and that the three formulas above hold. We note that if (32) holds then $\mu:=\llbracket \varphi \rrbracket v_{\varphi}\llcorner J(\varphi)$ is a ("finite") measure in $\mathcal{M}\left(\Omega, \boldsymbol{R}^{n}\right)$. Let us prove that $\mu$ is the weak derivative of $\varphi$, which will also prove $\varphi \in B V(\Omega, \boldsymbol{R})$. Thus, we have to prove that

$$
\int_{\Omega} \varphi \nabla f d \mathcal{V}=-\int_{J(\varphi)} f \llbracket \varphi \rrbracket d \mathcal{A}
$$

for every class-infinity function $f$ with support in $\Omega$. The application of the GaussGreen theorem to each of the sets $\Omega_{\alpha}$ provides

$$
\int_{\Omega_{\alpha}} \varphi \nabla f d \mathcal{V} \equiv a_{\alpha} \int_{\Omega_{\alpha}} \nabla f d \mathcal{V}=a_{\alpha} \int_{\partial \Omega_{\alpha}} f v_{\alpha} d \mathcal{A} .
$$

Summing these equations over all $\alpha$ and using that $v_{\alpha}=-v_{\beta}$, one obtains (34) and hence we have $\varphi \in B V(\Omega, \boldsymbol{R})$, (33) and all the remaining assertions of the remark. The converse implication is proved by reversing the above arguments.

\section{The function $\Phi$}

The goal of this section is to prove that the functions defined in items (i)-(iv) of Theorem 2.3 coincide. We denote these functions by $\Phi_{1}, \Phi_{2}, \Phi_{3}$ and $\Phi_{4}$, respectively, and prove that they are the same by establishing the cycle of relations

$$
\Phi_{1} \geq \Phi_{2} \geq \Phi_{3} \geq \Phi_{4}=\Phi_{1} .
$$

5.1. Proposition. $\Phi_{1} \geq \Phi_{2}$. 
Proof. It is easy to show that $\Phi_{2}$ is a subadditive function. Thus, the definition of $\Phi_{1}$ gives the assertion.

The proof of the following lemma contains a construction of the central minimizing sequence $u_{k} \in \mathcal{C}(M)$ for Theorem 2.3(iii). This sequence will be defined as the superposition of (a slight modification of) the sequence of step deformations $s_{k}, k=1, \ldots$, defined on $Q$ by

$$
s_{k}(x)=k^{-1} a\langle k x \cdot b\rangle,
$$

$x \in Q$. Clearly, $\nabla s_{k}=0$, and in view of (27),

$$
s_{k}(x) \rightarrow a(x \cdot b) \quad \text { on } Q
$$

as $k \rightarrow \infty$. Thus, $s_{k}$ satisfies the boundary condition $s_{k}=w_{a \otimes b}$ on $\partial Q$ in the asymptotic sense; however, the definition of $\mathcal{C}(a \otimes b)$ requires the exact form of that boundary condition. For this reason, we have to slightly modify $s_{k}$ near the boundary $\partial Q$ without violating the equation $\nabla s_{k}=0$.

5.2. Lemma. If $M \in \operatorname{Lin}$ and $\left(a_{i}, b_{i}\right) \in \boldsymbol{D}_{n}, i=1, \ldots, m$, satisfy

$$
M=\sum_{i=1}^{m} a_{i} \otimes b_{i},
$$

then there exists a sequence $u_{k} \in \mathcal{C}(M), k=1, \ldots$, such that

$$
\limsup _{k \rightarrow \infty} \Psi\left(\mathrm{D}^{\mathrm{s}} u_{k}\right) \leq \sum_{i=1}^{m} \psi\left(a_{i}, b_{i}\right) .
$$

We refer to Remark 5.3 for a mild condition on the sequence $\left(a_{i}, b_{i}\right)$ that guarantees that the lim sup in (36) strengthens to lim and the inequality sign to the equality sign.

Proof. We shall first construct the sequence $u_{k}$ for the particular case when $M=$ $a \otimes b$ is a dyad and then superimpose the sequences corresponding to the dyads $a_{i} \otimes b_{i}, i=1, \ldots, m$, to obtain the general case. Thus, let $(a, b) \in \boldsymbol{D}_{n}$ and construct a sequence $u_{k} \in \mathcal{C}(a \otimes b), k=1, \ldots$, such that

$$
\lim _{k \rightarrow \infty} \Psi\left(\mathrm{D}^{\mathrm{s}} u_{k}\right)=\psi(a, b) .
$$

Introduce the sets

$$
C_{k}=\left(1-k^{-2}\right) Q, \quad L_{l}=\left(1-(l+1)^{-2}\right) Q \backslash\left(1-l^{-2}\right) Q,
$$

$k, l \in N$, and observe that

$$
Q=C_{k} \cup \bigcup_{l=k}^{\infty} L_{l}
$$


with mutually disjoint summands for any $k \in N$. Here the product $t S$ of a set $S \subset \boldsymbol{R}^{n}$ and a real number $t$ is defined by $t S=\{t x: x \in S\}$. Equation (39) presents a decomposition of $Q$ into the main set $C_{k}$, which is a large subset of $Q$ for large $k$, while $L_{k}, L_{k+1}, \ldots$ present infinitely many rectangular layers filling the gap $Q \backslash C_{k}$ and becoming more and more refined towards the boundary of $Q$.

We use these sets $C_{k}, L_{k}, L_{k+1}, \ldots$ to define a sequence of scalar functions $\varphi_{k}: Q \rightarrow \boldsymbol{R}, k=2, \ldots$, by

$$
\varphi_{k}(x)= \begin{cases}(k-1)^{-2}\left\langle(k-1)^{2} x \cdot b\right\rangle & \text { if } x \in C_{k}, \\ l^{-2}\left\langle l^{2} x \cdot b\right\rangle & \text { if } x \in L_{l} \text { for some } l \geq k .\end{cases}
$$

Let us use Remark 4.3 to prove that $\varphi_{k} \in B V(Q, \boldsymbol{R})$. Clearly, $\varphi_{k}$ is a piecewise constant function in the sense of that remark. Using (25a), one finds that $x \cdot b-1 \leq$ $\varphi_{k}(x) \leq x \cdot b$; hence, $\left|\varphi_{k}\right|$ is bounded on $Q$ and thus $\varphi_{k} \in L^{1}(Q, \boldsymbol{R})$. It remains to verify (32). Let us show that in the present case (32) reads

$$
\int_{J\left(\varphi_{k}\right)}\left|\llbracket \varphi_{k} \rrbracket\right| d \mathcal{A}<\infty
$$

where

$$
J\left(\varphi_{k}\right)=C_{k}^{\circ} \cup \bigcup_{l=k}^{\infty}\left(L_{l}^{\circ} \cup L_{l}^{\partial}\right)
$$

is the jump set, with

$$
\begin{aligned}
& C_{k}^{\circ}=\left\{x \in C_{k}: k^{2} x \cdot b \in Z\right\}, \\
& L_{l}^{\circ}=\left\{x \in L_{l}: l^{2} x \cdot b \in Z\right\}, \quad L_{l}^{\partial}=\left(1-l^{-2}\right) \partial Q,
\end{aligned}
$$

and on $J\left(\varphi_{k}\right)$

$$
\llbracket \varphi_{k} \rrbracket v_{\varphi_{k}}= \begin{cases}(k-1)^{-2} b & \text { on } C_{k}^{\circ}, \\ l^{-2} b & \text { on } L_{l}^{\circ} \text { where } l \geq k, \\ \eta_{l} v_{k} & \text { on } L_{l}^{\partial} \text { where } l \geq k\end{cases}
$$

is the jump and normal to the jump set, with

$$
\eta_{l}(x)=l^{-2}\left\langle l^{2} x \cdot b\right\rangle-(l-1)^{-2}\left\langle(l-1)^{2} x \cdot b\right\rangle
$$

and with $v_{k}$ denoting the outer normal to the scaled cube $\left(1-k^{-2}\right) Q$. Equations (42) and (45) follow from the identities given in Remark 4.3. One has to enumerate the regions of constancy of $\varphi_{k}$ in an arbitrary way to obtain the system of sets $\Omega_{\alpha}$, $\alpha=1, \ldots$, and use the formulas of that remark. The details are left to the reader. This establishes the equivalence of the inequalities (32) and (41). To prove that 
(41) really holds, one finds from (45) that

$$
\int_{J\left(u_{k}\right)}\left|\llbracket \varphi_{k} \rrbracket\right| d \mathcal{A}=(k-1)^{-2} \mathcal{A}\left(C_{k}^{\circ}\right)+\sum_{l=k}^{\infty} l^{-2} \mathcal{A}\left(L_{l}^{\circ}\right)+\sum_{l=k}^{\infty} \int_{L_{l}^{\partial}}\left|\eta_{l}\right| d \mathcal{A} .
$$

We estimate the terms $\mathcal{A}\left(C_{k}^{\circ}\right), \mathcal{A}\left(L_{l}^{\circ}\right)$ and $\int_{L_{l}^{\partial}}\left|\eta_{l}(x)\right| d \mathcal{A}$ as follows. First, prove that

$$
\begin{aligned}
\left|\mathcal{A}\left(C_{k}^{\circ}\right)-(k-1)^{2} \mathcal{L}^{n}\left(C_{k}\right)\right| & \leq 2 n, \\
\left|\mathcal{A}\left(L_{l}^{\circ}\right)-l^{2} \mathcal{L}^{n}\left(L_{l}\right)\right| & \leq 4 n
\end{aligned}
$$

and hence

$$
\mathcal{A}\left(C_{k}^{\circ}\right) \leq 2 n+(k-1)^{2} \mathcal{L}^{n}\left(C_{k}\right), \quad \mathcal{A}\left(L_{l}^{\circ}\right) \leq 4 n+l^{2} \mathcal{L}^{n}\left(L_{l}\right) .
$$

Let us prove (48b); the proof of (48a) is similar. Let $\omega: L_{l} \rightarrow \boldsymbol{R}$ be defined by

$$
\omega(x)=l^{2} x \cdot b-\left\langle l^{2} x \cdot b\right\rangle, \quad x \in L_{l} .
$$

Then $\omega \in B V\left(L_{l}, \boldsymbol{R}\right), \mathrm{D} \omega=l^{2} b-b \mathcal{A}\left\llcorner L_{l}^{\circ}\right.$, and hence the Gauss-Green theorem yields

$$
\mathrm{D} \omega\left(L_{l}\right)=l^{2} \mathcal{L}^{n}\left(L_{l}\right) b-b \mathcal{A}\left(L_{l}^{\circ}\right)=\int_{\partial L_{l}} \omega v_{L_{l}} d \mathcal{A},
$$

from which

$$
\left|m \mathcal{L}^{n}\left(L_{l}\right)-\mathcal{A}\left(L_{l}^{\circ}\right)\right| \leq \int_{\partial L_{l}}|\omega| d \mathcal{A}
$$

We now observe that $|\omega| \leq 1$ on $\partial L_{l}$ and $\partial L_{l}=L_{l+1}^{\partial} \cup L_{l}^{\partial}$. Thus,

$$
\int_{\partial L_{l}}|\omega| d \mathcal{A} \leq \mathcal{A}\left(L_{l+1}^{\partial}\right)+\mathcal{A}\left(L_{l}^{\partial}\right) \leq 4 n
$$

since, elementarily, $\mathcal{A}\left(L_{l+1}^{\partial}\right) \leq 2 n$ and $\mathcal{A}\left(L_{l}^{\partial}\right) \leq 2 n$. Thus, we have (48b). Next prove that

$$
\left|\eta_{l}(x)\right| \leq 2(l-1)^{-2} \quad \text { on } L_{l}^{\partial} .
$$

Indeed, writing

$$
\left|\eta_{l}(x)\right|=\left|\left(l^{-2}\left\langle l^{2} x \cdot b\right\rangle-x \cdot b\right)-\left((l-1)^{-2}\left\langle(l-1)^{2} x \cdot b\right\rangle-x \cdot b\right)\right|,
$$

using the triangle inequality and the inequality (26) twice, with $t=x \cdot b$ and $k=l^{2}$ and $k=(l-1)^{2}$, one obtains

$$
\left|\eta_{l}(x)\right| \leq l^{-2}+(l-1)^{-2} \leq 2(l-1)^{-2}
$$

and hence

$$
\int_{L_{l}^{\partial}}\left|\eta_{l}\right| d \mathcal{A} \leq 2(l-1)^{-2} \mathcal{A}\left(L_{l}^{\partial}\right) \leq 4 n(l-1)^{-2} .
$$


The estimates (49) and (51) and the formula (47) provide

$$
\begin{aligned}
\int_{J\left(u_{k}\right)}\left|\llbracket \varphi_{k} \rrbracket\right| d \mathcal{A} & \leq 2 n(k-1)^{-2}+\mathcal{L}^{n}\left(C_{k}\right) \\
& +\sum_{l=k}^{\infty}\left(4 n l^{-2}+\mathcal{L}^{n}\left(L_{l}\right)\right)+\sum_{l=k}^{\infty} 4 n(l-1)^{-2} \\
& \leq 1+2 n(k-1)^{-2}+8 n \sum_{l=k}^{\infty}(l-1)^{-2}<\infty,
\end{aligned}
$$

where we have used

$$
\mathcal{L}^{n}\left(C_{k}\right)+\sum_{l=k}^{\infty} \mathcal{L}^{n}\left(L_{l}\right)=\mathcal{L}^{n}(Q)=1 .
$$

Thus, we have (41); hence, $\varphi_{k} \in B V(\Omega, \boldsymbol{R})$ for every $k$ and

$$
\mathrm{D} \varphi_{k}=\llbracket \varphi_{k} \rrbracket v_{\varphi_{k}} \mathcal{A}\left\llcorner J\left(\varphi_{k}\right)\right.
$$

and

$$
\nabla \varphi_{k}=0
$$

Finally, note that the boundary trace $\varphi_{k}^{\partial}$ of $\varphi_{k}$ on $\partial Q$ satisfies

$$
\varphi_{k}^{\partial}(x)=x \cdot b \quad \text { for every } x \in \partial Q .
$$

While a rigorous proof of this can be given by using the essential limit of $\varphi_{k}$ at $x \in Q$, we here only note that the definition of $\varphi_{k}$ yields that

$$
\lim _{j \rightarrow \infty} \varphi_{k}\left(x_{j}\right)=x \cdot b
$$

for any $x \in \partial Q$ and any sequence $x_{j} \in Q$ converging to $x$. For this it suffices to note that in view of (39) one finds that $x_{j}$ must belong to some $L_{l}$ for some $l=l(j) \geq k$. The limit $x_{j} \rightarrow x$ then implies that $l(k) \rightarrow \infty$, and then the definition (40) and the formula (27) provide (54).

We define the sequence $u_{k}: Q \rightarrow \boldsymbol{R}^{n}, k=2, \ldots$, by

$$
u_{k}(x)=a \varphi_{k}(x)
$$

for every $x \in Q$. By $\varphi_{k} \in S B V(Q, \boldsymbol{R})$ and by (52) and (53), we have $u_{k} \in \mathcal{C}(a \otimes b)$. Further, $\llbracket u_{k} \rrbracket=\llbracket \varphi_{k} \rrbracket a \otimes v_{\varphi_{k}} ;$ consequently, by (45),

$$
\psi\left(\llbracket u_{k} \rrbracket, v_{u_{k}}\right)= \begin{cases}(k-1)^{-2} \psi(a, b) & \text { on } C_{k}^{\circ}, \\ l^{-2} \psi(a, b) & \text { on } L_{l}^{\circ} \text { for any } l \geq k, \\ \psi\left(\eta_{l} a, v_{l}\right) & \text { on } L_{l}^{\partial} \text { for any } l \geq k\end{cases}
$$


and hence

$$
\Psi\left(\mathrm{D}^{\mathrm{s}} u_{k}\right)=\int_{J\left(u_{k}\right)} \psi\left(\llbracket u_{k} \rrbracket, v_{u_{k}}\right) d \mathcal{A}=(k-1)^{-2} \psi(a, b) \mathcal{A}\left(C_{k}^{\circ}\right)+\rho_{k},
$$

where

$$
\rho_{k}=\sum_{l=k}^{\infty} l^{-2} \psi(a, b) \mathcal{A}\left(L_{l}^{\circ}\right)+\sum_{l=k}^{\infty} \int_{L_{l}^{\partial}} \psi\left(\eta_{l} a, v_{l}\right) d \mathcal{A} .
$$

Dividing (48a) by $(k-1)^{2}$, we obtain

$$
(k-1)^{-2} \mathcal{A}\left(C_{k}^{\circ}\right) \rightarrow 1
$$

since $\mathcal{L}^{n}\left(C_{k}\right) \rightarrow 1$. Using (6), we obtain that the nonnegative number $\rho_{k}$ is bounded by (a constant multiple of) the quantity

$$
\begin{aligned}
d_{k} & =\sum_{l=k}^{\infty} l^{-2} \mathcal{A}\left(L_{l}^{\circ}\right)+\sum_{l=k}^{\infty} \int_{L_{l}^{\partial}}\left|\eta_{l}\right| d \mathcal{A} \\
& \leq \sum_{l=k}^{\infty} \mathcal{L}^{n}\left(L_{l}\right)+2 n(k-1)^{-2}+4 n \sum_{l=k}^{\infty}(l-1)^{-2} \\
& \leq k^{-2}+2 n(k-1)^{-2}+4 n \sum_{l=k}^{\infty}(l-1)^{-2}
\end{aligned}
$$

and hence $\rho_{k} \rightarrow 0$. Equations (55) and (56) then yield (37).

We now complete the proof in the general case. By the preceding part of the proof, for each $i \in\{1, \ldots, m\}$, there exists a sequence $u_{k}^{i} \in \mathcal{C}\left(a_{i} \otimes b_{i}, 0\right), k=1, \ldots$, such that

$$
\Psi\left(\mathrm{D}^{\mathrm{s}} u_{k}^{i}\right) \rightarrow \psi\left(a_{i}, b_{i}\right)
$$

as $k \rightarrow \infty$. Define $u_{k}:=\sum_{i=1}^{m} u_{k}^{i}$ for every $k$. By (28),

$$
\Psi\left(\mathrm{D}^{\mathrm{s}} u_{k}\right) \leq \sum_{i=1}^{m} \Psi\left(\mathrm{D}^{\mathrm{s}} u_{k}^{i}\right)
$$

Hence,

$$
\limsup _{k \rightarrow \infty} \Psi\left(\mathrm{D}^{\mathrm{s}} u_{k}\right) \leq \lim _{k \rightarrow \infty} \sum_{i=1}^{m} \Psi\left(\mathrm{D}^{\mathrm{s}} u_{k}^{i}\right)=\sum_{i=1}^{m} \psi\left(a_{i}, b_{i}\right)
$$

by (57).

5.3. Remark. If the sequence $\left(a_{i}, b_{i}\right)$ satisfies the condition

$$
b_{i} \neq b_{j} \quad \text { and } \quad b_{i} \neq-b_{j} \quad \text { whenever } 1 \leq i<j \leq m,
$$


then the sequence $u_{k}$ can be chosen as to satisfy, instead of the inequality (36), the equality

$$
\lim _{k \rightarrow \infty} \Psi\left(\mathrm{D}^{\mathrm{s}} u_{k}\right)=\sum_{i=1}^{m} \psi\left(a_{i}, b_{i}\right) .
$$

Indeed, the inspection of the proof of Lemma 5.2 shows that the source of the inequality (36) is the subadditivity in (58) which cannot be replaced by the equality unless the discontinuity sets $J\left(u_{i}\right)$ pairwise intersect on a set of null $\mathcal{A}$ measure (see Proposition 4.1). Condition (59) guarantees that. However, inequality (36) suffices for our purposes.

5.4. Proposition. $\Phi_{2} \geq \Phi_{3} \geq \Phi_{4}$.

Proof. To prove $\Phi_{2} \geq \Phi_{3}$, we take any sequence $\left(a_{i}, b_{i}\right) \in \boldsymbol{D}_{n}, i=1, \ldots, m$, such that $\sum_{i=1}^{m} a_{i} \otimes b_{i}=M$ and consider the infimum as in the definition of $\Phi_{2}$ in (16). Hence, for the given sequence $\left(a_{i}, b_{i}\right) \in \boldsymbol{D}_{n}$, we construct a sequence of maps $u_{k} \in \mathcal{C}(M), k=1, \ldots$, as in Lemma 5.2. Then

$$
\Phi_{3}(M) \leq \Psi\left(\mathrm{D}^{\mathrm{s}} u_{k}\right)
$$

by the definition of $\Phi_{3}$. Letting $k \rightarrow \infty$ and using (36), we obtain

$$
\Phi_{3}(M) \leq \sum_{i=1}^{m} \psi\left(a_{i}, b_{i}\right) .
$$

Taking the infimum over all sequences $a_{i}$ and $b_{i}$, one obtains from the definition of $\Phi_{2}$ the inequality $\Phi_{3}(M) \leq \Phi_{2}(M)$. The inequality $\Phi_{3} \geq \Phi_{4}$ is immediate.

5.5. Proposition. $\Phi_{4}=\Phi_{1}$.

Proof. We seek to prove that $\Phi_{4}$ is the biggest subadditive function satisfying $\Phi_{4}(a \otimes b) \leq \psi(a, b)$ for any $(a, b) \in \boldsymbol{D}_{n}$. To prove the subadditivity of $\Phi_{4}$, let $A, B \in$ Lin and $u \in \mathcal{B}(A)$ and $v \in \mathcal{B}(B)$. Proposition 4.1 and (17) yield $u+v \in$ $\mathcal{B}(A+B)$ and

$$
\Phi_{4}(A+B) \leq \Psi\left(\mathrm{D}^{\mathrm{s}} u+\mathrm{D}^{\mathrm{s}} v\right) \leq \Psi\left(\mathrm{D}^{\mathrm{s}} u\right)+\Psi\left(\mathrm{D}^{\mathrm{s}} v\right) .
$$

Taking the infimum over all $u$ and $v$ then gives the subadditivity

$$
\Phi_{4}(A+B) \leq \Phi_{4}(A)+\Phi_{4}(B) .
$$

Next we note that the biggest subadditive function $\Theta$ such that

$$
\Theta(a \otimes b) \leq \psi(a, b)
$$

for any $(a, b) \in \boldsymbol{D}_{n}$ is automatically positively homogeneous; thus, it suffices to prove the maximality of $\Phi_{4}$ among all subadditive and positively homogeneous functions satisfying (60). Thus, let $\Theta$ be such a function and let $M \in \operatorname{Lin}$ and 
$u \in \mathcal{B}(M)$. Then by (60) and by Jensen's inequality for positively homogeneous subadditive functions,

$$
\begin{aligned}
\Psi\left(\mathrm{D}^{\mathrm{s}} u\right) & :=\int_{J(u)} \psi\left(\llbracket u \rrbracket, v_{u}\right) d \mathcal{A} \\
& \geq \int_{J(u)} \Theta\left(\llbracket u \rrbracket \otimes v_{u}\right) d \mathcal{A} \\
& \geq \Theta\left(\int_{J(u)} \llbracket u \rrbracket \otimes v_{u} d \mathcal{A}\right) .
\end{aligned}
$$

We now combine the boundary condition $u=w_{M}$ on $\partial Q$ and relation $\int_{Q} \nabla u d \mathcal{L}^{n}=0$ with the Gauss-Green theorem to obtain

$$
\begin{aligned}
\int_{J(u)} \llbracket u \rrbracket \otimes v_{u} d \mathcal{A} & =\int_{J(u)} \llbracket u \rrbracket \otimes v_{u} d \mathcal{A}+\int_{Q} \nabla u d \mathcal{L}^{n} \\
& =\int_{Q} 1 d \mathrm{D} u \\
& =\int_{\partial Q} M x \otimes v_{Q} d \mathcal{A}=M .
\end{aligned}
$$

Thus, (61) yields

$$
\Psi\left(\mathrm{D}^{\mathrm{s}} u\right) \geq \Theta(M) .
$$

Taking the infimum over all $u \in \mathcal{B}(M)$, we obtain $\Phi_{4}(M) \geq \Theta(M)$.

This proves $\Phi_{1}=\Phi_{2}=\Phi_{3}=\Phi_{4}$. We define the function $\Phi$ by $\Phi=\Phi_{1}$.

\section{Completion of the proof of Theorem 2.3}

For this section, we put, for every $(a, b) \in \boldsymbol{D}_{n}$,

$$
\mathrm{C}(a, b):=\left\{u \in \operatorname{SBV}\left(Q_{b}\right): u=z_{a, b} \text { on } \partial Q_{b}, \nabla u=0 \text { on } Q_{b}\right\}
$$

and denote by $\Phi_{5}(a, b)$ the infimum in (19). We then extend $\Phi_{5}$ to $\boldsymbol{R}^{n} \times \boldsymbol{R}^{n}$ by homogeneity in the second variable.

6.1. Proposition. We have $H(A, B)=\Phi(A-B)$ for every $A, B \in$ Lin.

Proof. We employ Theorem 2.2 and the definition of $\Phi$ in (18). Invoking (11), we take any $u \in S B V(Q)$ satisfying $u=w_{A}$ on $\partial Q$, and $\int_{Q} \nabla u d \mathcal{L}^{n}=B$. Then $v$, given by $v(x)=u(x)-B x, x \in Q$, satisfies $v \in \mathcal{B}(A-B)$ and $\Psi\left(\mathrm{D}^{\mathrm{s}} u\right)=\Psi\left(\mathrm{D}^{\mathrm{s}} v\right)$.

6.2. Lemma. We have $\Phi_{5}(a, b) \leq \Phi(a \otimes b)$ for every $(a, b) \in \boldsymbol{D}_{n}$.

Proof. Let $(a, b) \in \boldsymbol{D}_{n}$, and let $\left(a_{i}, b_{i}\right) \in \boldsymbol{D}_{n}, i=1, \ldots, m$, be a sequence satisfying

$$
a \otimes b=\sum_{i=1}^{m} a_{i} \otimes b_{i} .
$$


Our goal is to construct a sequence $u_{k} \in \mathrm{C}(a, b), k=1, \ldots$, such that

$$
\limsup _{k \rightarrow \infty} \int_{J\left(u_{k}\right)} \psi\left(\llbracket u_{k} \rrbracket, v_{u_{k}}\right) d \mathcal{A} \leq \sum_{i=1}^{m} \psi\left(a_{i}, b_{i}\right) .
$$

To define $u_{k}$, let

$$
P=\left\{x \in \boldsymbol{R}^{n}: x \cdot b=0\right\}
$$

be the plane through the origin perpendicular to $b$, let $\Pi$ be the projection from $\boldsymbol{R}^{n}$ onto $P$, let

$$
F=P \cap Q_{b},
$$

and put

$$
B_{k}=\left\{x \in \boldsymbol{R}^{n}: \Pi(x) \in\left(1-k^{-1}\right) F, 0 \leq x \cdot b<k^{-1}\right\}
$$

for any $k \in N$. Define $u_{k}: Q_{b} \rightarrow \boldsymbol{R}^{n}$ by

$$
u_{k}(x)= \begin{cases}v_{k}(x) & \text { if } x \in B_{k}, \\ z_{a, b}(x) & \text { else, }\end{cases}
$$

$x \in Q_{b}$, where

$$
v_{k}(x)=\sum_{i=1}^{m} k^{-1} a_{i}\left\langle k^{2} x \cdot b_{i}\right\rangle \quad \text { for any } x \in \boldsymbol{R}^{n} \text { and } k \in N .
$$

Employing Remark 4.2, we see that $u_{k} \in S B V\left(Q_{b}\right)$; furthermore, clearly, $u_{k}=z_{a, b}$ on $\partial Q_{b}$ and $\nabla u_{k}=0$ on $Q_{b}$; hence, $u_{k} \in \mathrm{C}(a, b)$.

We proceed to prove (63). We have

$$
J\left(u_{k}\right)=N_{k} \cup M_{k} \cup L_{k} \cup S_{k},
$$

where

$$
\begin{aligned}
N_{k} & =F \backslash\left(1-k^{-1}\right) F, \\
M_{k} & =\left\{x \in \partial B: 0<x \cdot b<k^{-1}\right\}, \\
S_{k} & =\left\{x \in \boldsymbol{R}^{n}: \Pi(x) \in\left(1-k^{-1}\right) F, x \cdot b=k^{-1}\right\}, \\
L_{k} & =\bigcup_{i=1}^{m} L_{k}^{i} \quad \text { where } L_{k}^{i}=\left\{x \in B_{k}: k^{2} x \cdot b_{i} \in Z\right\} .
\end{aligned}
$$

The jump of $u_{k}$ and the normal to the jump set are

$$
\llbracket u_{k} \rrbracket(x) v_{u_{k}}(x)= \begin{cases}k^{-1} \sum_{i=1}^{m} a_{i} \otimes b_{i} 1_{L_{k}^{i}}(x) & \text { if } x \in L_{k}, \\ a \otimes b & \text { if } x \in N_{k}, \\ \left(a-v_{k}(x)\right) \otimes v_{k} & \text { if } x \in M_{k}, \\ \left(a-v_{k}(x)\right) \otimes b & \text { if } x \in S_{k},\end{cases}
$$


$x \in J\left(u_{k}\right)$, where $v_{k}$ is the outer normal to $B_{k}$ and $1_{L_{k}^{i}}$ is the characteristic function of the set $L_{k}^{i}$. Hence, the subadditivity of $\psi$ in the first variable yields

$$
\int_{L_{k}} \psi\left(\llbracket u_{k} \rrbracket, v_{u_{k}}\right) d \mathcal{A} \leq k^{-1} \sum_{i=1}^{m} \psi\left(a_{i}, b_{i}\right) \mathcal{A}\left(L_{k}^{i}\right) ;
$$

consequently

$$
\begin{aligned}
\int_{J\left(u_{k}\right)} \psi\left(\llbracket u_{k} \rrbracket, v_{u_{k}}\right) d \mathcal{A} \leq k^{-1} \sum_{i=1}^{m} \psi\left(a_{i}, b_{i}\right) \mathcal{A}( & \left.L_{k}^{i}\right)+\psi(a, b) \mathcal{A}\left(N_{k}\right) \\
& \left.+\int_{M_{k}} \psi\left(a-v_{k}(x)\right), v_{k}\right) d \mathcal{A} \\
& \left.+\int_{S_{k}} \psi\left(a-v_{k}(x)\right), b\right) d \mathcal{A} .
\end{aligned}
$$

Let us now analyze the terms on the right-hand side of (67). Using the considerations as in the proof of Lemma 5.2 (see (48) and (49)), one finds that

$$
k^{-1} \mathcal{A}\left(L_{k}^{i}\right) \rightarrow 1
$$

as $k \rightarrow \infty$ for every $i=1, \ldots, m$. Thus,

$$
k^{-1} \sum_{i=1}^{m} \psi\left(a_{i}, b_{i}\right) \mathcal{A}\left(L_{k}^{i}\right) \rightarrow \sum_{i=1}^{m} \psi\left(a_{i}, b_{i}\right) .
$$

Further,

$$
\psi(a, b) \mathcal{A}\left(N_{k}\right) \rightarrow 0
$$

since, obviously,

$$
\mathcal{A}\left(N_{k}\right) \rightarrow 0
$$

Next note that, by (62) and (26),

$$
\begin{aligned}
\left|k a(x \cdot b)-v_{k}(x)\right| & =\left|k a(x \cdot b)-\sum_{i=1}^{m} k^{-1} a_{i}\left\langle k^{2} x \cdot b_{i}\right\rangle\right| \\
& =\left|k \sum_{i=1}^{m} a_{i}\left(x \cdot b_{i}\right)-k^{-2} a_{i}\left\langle k^{2} x \cdot b_{i}\right\rangle\right| \\
& \leq\left|k \sum_{i=1}^{m}\right| a_{i}||\left(x \cdot b_{i}\right)-k^{-2}\left\langle k^{2} x \cdot b_{i}\right\rangle|| \\
& \leq k^{-1} \sum_{i=1}^{m}\left|a_{i}\right| .
\end{aligned}
$$


Then if $x \in M_{k}$,

$$
\begin{aligned}
\left|a-v_{k}(x)\right| & \leq|a-k a(x \cdot b)|+\left|k a(x \cdot b)-v_{k}(x)\right| \\
& \leq|a|+k|a||x \cdot b|+k^{-1} \sum_{i=1}^{m}\left|a_{i}\right| \\
& \leq|a|+|a|+k^{-1} \sum_{i=1}^{m}\left|a_{i}\right|
\end{aligned}
$$

since $k|x \cdot b| \leq 1$ on $M_{k}$. Thus, $\left|a-v_{k}(x)\right| \leq c<\infty$ for any $x \in M_{k}$ and any $k=1, \ldots$ A combination with $(6)$ and

$$
\mathcal{A}\left(M_{k}\right) \rightarrow 0
$$

then provides

$$
\left.\int_{M_{k}} \psi\left(a-v_{k}(x)\right), v_{k}\right) d \mathcal{A} \rightarrow 0 .
$$

Similarly, if $x \in S_{k}$, then $k x \cdot b=1$ and hence

$$
\left|a-v_{k}(x)\right| \leq\left|k a(x \cdot b)-v_{k}(x)\right| \leq k^{-1} \sum_{i=1}^{m}\left|a_{i}\right| \rightarrow 0 .
$$

Thus, (6) yields

$$
\left.\int_{S_{k}} \psi\left(a-v_{k}(x)\right), b\right) d \mathcal{A} \rightarrow 0
$$

since $\mathcal{A}\left(S_{k}\right) \leq 1$ for all $k$. Consequently, a combination of (67) with (68)-(71) provides (63) and hence the definition of $\Phi_{5}$ gives

$$
\Phi_{5}(a, b) \leq \sum_{i=1}^{m} \psi\left(a_{i}, b_{i}\right)
$$

for any sequence $\left(a_{i}, b_{i}\right)$ satisfying (62). Taking the infimum of the right-hand side over all such sequences and using the definition of $\Phi_{2} \equiv \Phi$, we obtain the assertion.

6.3. Lemma. We have $\Phi_{5}(a, b) \geq \Phi(a \otimes b)$ for every $(a, b) \in \boldsymbol{D}_{n}$.

Proof. Let $u \in \mathrm{C}(a, b)$. Then, by Jensen's inequality,

$$
\begin{aligned}
\int_{J(u)} \psi\left(\llbracket u \rrbracket, v_{u}\right) d \mathcal{A} & \geq \int_{J(u)} \Phi\left(\llbracket u \rrbracket \otimes v_{u}\right) d \mathcal{A} \\
& \geq \Phi\left(\int_{J(u)} \llbracket u \rrbracket \otimes v_{u} d \mathcal{A}\right) \\
& =\Phi(a \otimes b)
\end{aligned}
$$


since the boundary condition $u=z_{a, b}$ on $\partial Q_{b}$ implies

$$
\int_{J(u)} \llbracket u \rrbracket \otimes v_{u} d \mathcal{A}=a \otimes b .
$$

That is, we have

$$
\int_{J(u)} \psi\left(\llbracket u \rrbracket, v_{u}\right) d \mathcal{A} \geq \Phi(a \otimes b)
$$

for every $u \in \mathrm{C}(a, b)$. Taking the infimum, we obtain $\Phi_{5}(a, b) \geq \Phi(a \otimes b)$.

6.4. Proposition. We have $h(a, b)=\Phi(a \otimes b)$ for every $(a, b) \in \boldsymbol{D}_{n}$.

Proof. This follows immediately from (12) and (19).

This completes the proof of Theorem 2.3.

\section{Derivation of the examples}

Derivation of Example 2.5 and (21). We consider $\psi_{|\cdot|}(a, b)=|a \cdot b|$ first and prove (21a). Clearly, the function $\Theta(M)=|\operatorname{tr} M|$ is a subadditive function satisfying (14) with $\psi=\psi_{|\cdot|}$ and hence (16) gives $\Phi_{|\cdot|}(M) \geq|\operatorname{tr} M|$ for any $M \in$ Lin. To prove the opposite inequality, we note that the definition (15) of $\Phi_{|\cdot|}$ gives

$$
\psi_{|\cdot|}(a, b)=\Theta(a \otimes b) \leq \Phi_{|\cdot|}(a \otimes b) \leq \psi_{|\cdot|}(a, b)
$$

for every $(a, b) \in \boldsymbol{D}_{n}$ and hence

$$
\Phi_{|\cdot|}(a \otimes b)=|a \cdot b| \quad \text { and in particular } \quad \Phi_{|\cdot|}(a \otimes b)=0 \quad \text { if } a \cdot b=0,
$$

which determines $\Phi_{|\cdot|}$ on tensor products $a \otimes b$. As a consequence, if $N \in$ Lin can be written as

$$
N=\sum_{i=1}^{m} a_{i} \otimes b_{i}
$$

where $\left(a_{i}, b_{i}\right) \in \boldsymbol{R}^{n} \times \boldsymbol{R}^{n}, i=1, \ldots, m$, where

$$
a_{i} \cdot b_{i}=0 \text { for all } i=1, \ldots, m,
$$

then $\Phi_{|\cdot|}(N)=0$ since

$$
0 \leq \Phi_{|\cdot|}(N) \leq \sum_{i=1}^{m} \Phi_{|\cdot|}\left(a_{i} \otimes b_{i}\right) \leq \sum_{i=1}^{m} \psi\left(a_{i}, b_{i}\right)=\sum_{i=1}^{m}\left|a_{i} \cdot b_{i}\right|=0 .
$$

To determine $\Phi_{|\cdot|}$ on a general $M \in$ Lin, we write $M=A+W$ where $A$ and $W$ are the symmetric and skew parts of $M$. Let $e_{1}, \ldots, e_{n}$ be an orthonormal basis of eigenvectors of $A$ with the eigenvalues $\lambda_{i}$; hence, $A=\sum_{i=1}^{n} \lambda_{i} e_{i} \otimes e_{i}$. Then

$$
M=B+N
$$


where

$$
\begin{aligned}
& B=(\operatorname{tr} M) e_{1} \otimes e_{1}, \\
& N=W+\sum_{i=2}^{n} \lambda_{i}\left(e_{i} \otimes e_{1}-e_{1} \otimes e_{i}-\left(e_{1}+e_{i}\right) \otimes\left(e_{1}-e_{i}\right)\right) .
\end{aligned}
$$

Since $W$ is a linear combination of the dyads $e_{i} \otimes e_{j}, 1 \leq i \neq j \leq n$, one sees that $N$ is of the form (72)-(73) and hence $\Phi_{|\cdot|}(N)=0$; consequently

$$
\Phi_{|\cdot|}(M) \leq \Phi_{|\cdot|}(B)+\Phi_{|\cdot|}(N)=\Phi_{|\cdot|}(B)=\psi\left((\operatorname{tr} M) e_{1}, e_{1}\right)=|\operatorname{tr} M| .
$$

Equations 13 complete the proof of (21a).

To prove the two equations in (21b), we employ (21a) and (21b) as follows. One has $\psi_{ \pm}(a, b)=\frac{1}{2}(|a \cdot b| \pm a \cdot b)$, and hence, if $\left(a_{i}, b_{i}\right) \in \boldsymbol{D}_{n}$ and $M \in$ Lin satisfy $\sum_{i=1}^{m} a_{i} \otimes b_{i}=M$, then

$$
\sum_{i=1}^{m} \psi_{ \pm}\left(a_{i}, b_{i}\right)=\frac{1}{2}\left(\sum_{i=1}^{m} \psi_{|\cdot|}\left(a_{i}, b_{i}\right) \pm \operatorname{tr} M\right) .
$$

Taking the infimum as in (16) and using the above evaluation of $\Phi_{|\cdot|}$ gives

$$
\Phi_{ \pm}(M)=\frac{1}{2}\left(\Phi_{|\cdot|}(M) \pm \operatorname{tr} M\right)=\frac{1}{2}(|\operatorname{tr} M| \pm \operatorname{tr} M)=\{\operatorname{tr} M\}_{ \pm},
$$

which is (21b).

Derivation of Example 2.6 and (23). The function $\Theta(M)=\left|M^{\mathrm{T}} p\right|$ is a subadditive function satisfying (14), and we obtain in the same way as in the proof of Example 2.5 that $\Phi(M) \geq\left|M^{\mathrm{T}} p\right|$ for any $M \in \operatorname{Lin}$ and

$$
\Phi(a \otimes b)=|a \cdot p| \quad \text { and in particular } \quad \Phi(a \otimes b)=0 \quad \text { if } a \cdot p=0 .
$$

To prove $\Phi(M) \leq\left|M^{\mathrm{T}} p\right|$, we assume without loss of generality that $|p|=1$ and let $\left\{p, e_{2}, \ldots e_{n}\right\}$ be any orthonormal basis. In view of $\mathbf{1}=p \otimes p+\sum_{i=2}^{n} e_{i} \otimes e_{i}$,

$$
M=\mathbf{1} M=p \otimes M^{\mathrm{T}} p+\sum_{i=2}^{n} e_{i} \otimes M^{\mathrm{T}} e_{i} ;
$$

normalizing the second members of the dyads, we obtain

$$
M=\left|M^{\mathrm{T}} p\right| p \otimes \operatorname{sgn}\left(M^{\mathrm{T}} p\right)+\sum_{i=2}^{n}\left|M^{\mathrm{T}} e_{i}\right| e_{i} \otimes \operatorname{sgn}\left(M^{\mathrm{T}} e_{i}\right) .
$$

The subadditivity of $\Phi$ provides

$$
\Phi(M) \leq \Phi\left(\left|M^{\mathrm{T}} p\right| p \otimes \operatorname{sgn}\left(M^{\mathrm{T}} p\right)\right)+\sum_{i=2}^{n} \Phi\left(\left|M^{\mathrm{T}} e_{i}\right| e_{i} \otimes \operatorname{sgn}\left(M^{\mathrm{T}} e_{i}\right)\right)=\left|M^{\mathrm{T}} p\right|
$$

by (74). Thus, $\Phi(M) \leq\left|M^{\mathrm{T}} p\right|$ and the proof of (23) is complete. 


\section{Acknowledgments}

I thank David Owen for a detailed discussion on the previous version of this work. His stimulating questions contributed to the final version of the results presented here. Also, David Owen and the two anonymous reviewers pointed out to me that it is possible to replace my original coercivity assumption on $\psi$ in Assumption 2.1(ii) by mere nonnegativity, which is necessary to cover Examples 2.5 and 2.6 and which is possible by [Choksi and Fonseca 1997, Remark 3.3]. I am also thankful for the support of the institutional research plan RVO 67985840.

\section{References}

[Ambrosio et al. 2000] L. Ambrosio, N. Fusco, and D. Pallara, Functions of bounded variation and free discontinuity problems, Clarendon, New York, 2000.

[Baía et al. 2011] M. Baía, J. Matias, and P. M. Santos, "A survey on structured deformations", São Paulo J. Math. Sci. 5:2 (2011), 185-201.

[Baía et al. 2012] M. Baía, J. Matias, and P. M. Santos, "A relaxation result in the framework of structured deformations in a bounded variation setting", Proc. Roy. Soc. Edinburgh A 142:2 (2012), 239-271.

[Barroso et al. 2017] A. C. Barroso, J. Matias, M. Morandotti, and D. R. Owen, "Explicit formulas for relaxed disarrangement densities arising from structured deformations", Math. Mech. Complex Syst. 5:2 (2017), 163-189.

[Choksi and Fonseca 1997] R. Choksi and I. Fonseca, "Bulk and interfacial energy densities for structured deformations of continua”, Arch. Rational Mech. Anal. 138:1 (1997), 37-103.

[Del Piero 2001] G. Del Piero, “The energy of a one-dimensional structured deformation", Math. Mech. Solids 6:4 (2001), 387-408.

[Del Piero 2004] G. Del Piero, "Foundations of the theory of structured deformations", pp. 125-175 in Multiscale modeling in continuum mechanics and structured deformations, edited by G. Del Piero and D. R. Owen, International Centre for Mechanical Sciences 447, Springer, Vienna, 2004.

[Del Piero and Owen 1993] G. Del Piero and D. R. Owen, "Structured deformations of continua", Arch. Rational Mech. Anal. 124:2 (1993), 99-155.

[Del Piero and Owen 1995] G. Del Piero and D. R. Owen, "Integral-gradient formulae for structured deformations", Arch. Rational Mech. Anal. 131:2 (1995), 121-138.

[Del Piero and Owen 2004] G. Del Piero and D. R. Owen (editors), Multiscale modeling in continuum mechanics and structured deformations, International Centre for Mechanical Sciences 447, Springer, Vienna, 2004.

[Evans and Gariepy 1992] L. C. Evans and R. F. Gariepy, Measure theory and fine properties of functions, CRC, Boca Raton, FL, 1992.

[Federer 1969] H. Federer, Geometric measure theory, Grundlehren der math. Wissenschaften 153, Springer, Berlin, 1969.

[Hille and Phillips 1957] E. Hille and R. S. Phillips, Functional analysis and semi-groups, rev. ed., AMS Colloquium Publications 31, American Mathematical Society, Providence, RI, 1957.

[Owen and Paroni 2015] D. R. Owen and R. Paroni, "Optimal flux densities for linear mappings and the multiscale geometry of structured deformations", Arch. Ration. Mech. Anal. 218:3 (2015), $1633-1652$. 
[Reshetnyak 1968] Yu. G. Reshetnyak, "The weak convergence of completely additive vector-valued set functions”, Siberian Math. J. 9:6 (1968), 1039-1045.

[Šilhavý 2015] M. Šilhavý, "On the approximation theorem for structured deformations from $B V(\Omega)$ ", Math. Mech. Complex Syst. 3:1 (2015), 83-100.

[Ziemer 1989] W. P. Ziemer, Weakly differentiable functions: Sobolev spaces and functions of bounded variation, Graduate Texts in Math. 120, Springer, Berlin, 1989.

Received 25 Oct 2016. Revised 7 Mar 2017. Accepted 16 May 2017.

MIROSLAV ŠILHAVÝ: silhavy@math.cas.cz

Institute of Mathematics, Academy of Sciences of the Czech Republic, Žitná 25, 11567 Pragua 1, Czech Republic 